\title{
EQUIVALENCE OF SUMMATORY CONDITIONS ALONG SEQUENCES FOR BOUNDED HOLOMORPHIC FUNCTIONS
}

\author{
VLADIMIR YA. EIDERMAN PASCAL J. THOMAS
}

\begin{abstract}
A sequence of points $z_{k}$ in the unit disk is said to be thin for a given positive function $\rho$, if there is a nontrivial bounded holomorphic function such that the infinite series $\sum_{k} \rho\left(1-\left|z_{k}\right|\right)\left|f\left(z_{k}\right)\right|$ converges. All sequences will be assumed hyperbolically separated. We give necessary and sufficient conditions for the problem of thinness of a sequence to be non-trivial (one way or the other), and for two different positive functions $\rho_{1}, \rho_{2}$ to give rise to the same thin sequences. Along the way, some concrete conditions (necessary or sufficient) for a sequence to be thin are obtained.
\end{abstract}

This paper is dedicated to the memory of Matts Essén.

\section{INTRODUCTION AND MAIN RESULTS}

Let $\mathcal{F}$ be a certain class of holomorphic functions in a domain $G \subset \mathbb{C}$. N. Nikolski Ni] and S. Khavinson $\left[\mathrm{Kh}\right.$ established the existence of positive numbers $\left\{t_{k}\right\}$ (satisfying certain additional conditions) and of a sequence of points $\left\{z_{k}\right\} \subset G$ such that if $\sum_{k} t_{k}\left|f\left(z_{k}\right)\right|<\infty$, then $f \equiv 0$ for every $f \in \mathcal{F}$. Theorems of such kind arise in various applications: operator theory [Ni], approximation theory [Kh], E3], sampling, etc. They are also of independent interest.

We shall consider the case when $G$ is the unit disk $\mathbb{D}=\{|z|<1\},\left|z_{k}\right| \rightarrow 1$ as $k \rightarrow \infty$, and $\mathcal{F}$ is the class $H^{\infty}(\mathbb{D})$ of bounded holomorphic functions in $\mathbb{D}$. Note that since functions in the Nevanlinna class (also called "of bounded characteristic") can be written as the quotient of two bounded functions, all our results also apply automatically to that class. In [CTW], E1], E2], EE] sufficient conditions on $\left\{z_{k}\right\}$ were obtained in the case $t_{k} \equiv 1$. In [NPT, EE the problem of description of sequences $\left\{z_{k}\right\}$ was studied for $t_{k}=1-\left|z_{k}\right|$ (see [EE] for a more detailed survey).

In order to avoid summing repeatedly values of the function $f$ at nearby points, we impose a uniform condition of discreteness on our sequence, namely that it be separated in the pseudohyperbolic distance $d_{G}$.

Key words and phrases. functions of bounded characteristic, uniqueness, sampling .

Most of this paper was written while the first-named author was visiting Université Bordeaux I in the Fall of 2002, supported by CNRS. The first-named author thanks these institutions for their hospitality and support, and is especially grateful to Alexander Borichev who arranged this visit. Research of the first-named author is partially supported also by the Russian Foundation of Basic Research, Grant 01-01-00608, and by the Ministry of Education of Russia, Grant 00-1.0-199.

${ }^{0} 2000$ Mathematics Subject Classification 30D50 
Definition 1.1. We shall say that $\left\{z_{k}\right\}$ is separated, if

$$
\inf _{n \neq k} d_{G}\left(z_{j}, z_{k}\right):=\inf _{n \neq k}\left|\frac{z_{n}-z_{k}}{1-\bar{z}_{n} z_{k}}\right|=\delta>0 .
$$

A natural special case of the problem is that of radial coefficients: $t_{k}=\rho\left(1-\left|z_{k}\right|\right)$, where $\rho(t), t \in(0,1)$, is a positive function. It will be convenient to write the function $\rho(t)$ in the form $\rho(t)=\rho_{\theta}(t)=t e^{\theta(t)}$.

Definition 1.2. A sequence $\left\{z_{k}\right\}$ is said to be $\theta$-thin, if there exists a function $f \in H^{\infty}$ such that $f \not \equiv 0$ and

$$
\sum_{k} \rho_{\theta}\left(1-\left|z_{k}\right|\right)\left|f\left(z_{k}\right)\right|=\sum_{k}\left(1-\left|z_{k}\right|\right) e^{\theta\left(1-\left|z_{k}\right|\right)}\left|f\left(z_{k}\right)\right|<\infty .
$$

A non- $\theta$-thin sequence is said to be $\theta$-thick. The class of $\theta$-thin separated sequences is denoted by $\Lambda_{\theta}$.

Obviously, $\Lambda_{\theta_{1}} \subset \Lambda_{\theta_{2}}$ for $\theta_{1}>\theta_{2}$.

Our main results (Theorem 1.4 and Proposition [1.6) determine which pairs of functions $\theta_{1}, \theta_{2}$ give rise to the same class of $\theta$-thin separated sequences. Those results exhibit a certain hierarchy among separated sequences in the disk. In particular, the classes $\Lambda_{\theta}$ corresponding to $\rho(t)=t^{\alpha}, 0 \leq \alpha<1$, are all the same, but the case $\rho(t)=t$ is distinct. Before the beginning of the spectrum (for sufficiently big $\rho$, in particular all such $\rho$ must verify $\lim \sup _{t \rightarrow 0} \rho(t)=\infty$ ) all separated non Blaschke sequences are thick (Theorem 1.3). After the other end of the spectrum (for "small" $\rho$, in particular $\liminf _{t \rightarrow 0} \rho(t) / t=0$ for all such $\rho$ ) all separated sequences (even maximal separated sequences, or "nets") are thin (Proposition 1.5).

Our first statement is about the set of all possible positive functions $\theta$.

Theorem 1.3. Suppose that $\theta(t)$ is a positive continuous nonincreasing function. There exists a non Blaschke separated $\theta$-thin sequence $\left\{z_{k}\right\}$ if and only if

$$
\int_{0} \frac{d t}{t \theta(t)}=\infty
$$

Obviously, Theorem 1.3 implies the existence of $\theta$-thin sequences for all negative functions $\theta(t)$.

Theorem 1.4. a) If $t_{1}, t_{2}$ are positive functions and $\theta_{1}(t) \approx \theta_{2}(t)$, then $\Lambda_{\theta_{1}}=\Lambda_{\theta_{2}}$.

b) If $\theta_{1}(t) / \theta_{2}(t) \rightarrow \infty$ as $t \rightarrow 0$ and $\theta_{2}(t)$ is a positive continuous nonincreasing function satisfying (1.3), then $\Lambda_{\theta_{1}} \neq \Lambda_{\theta_{2}}$, i. e. there exists a sequence $\left\{z_{k}\right\}$ such that $\left\{z_{k}\right\} \in \Lambda_{\theta_{2}}$, but $\left\{z_{k}\right\} \notin \Lambda_{\theta_{1}}$.

That (1.3) is a sufficient condition for the existence of elements in $\Lambda_{\theta}$ will follow from part (b) of this result, applied to $\theta_{2}=\theta$. The necessity part in Theorem 1.3 will be proved in Section 2. Theorem 1.4 will be proved in Section [3,

Consider now functions $\theta$ bounded above (including negative ones).

Proposition 1.5. Assume that $\rho_{\theta}(t)$ is a continuous nondecreasing function such that $\rho_{\theta}(t) \leq C t$ with $C>0$. There exists a separated $\theta$-thick sequence $\left\{z_{k}\right\}$ if and only if

$$
\int_{0} \frac{\rho_{\theta}(t) d t}{t^{2}}=\infty, \quad \rho_{\theta}(t)=t e^{\theta(t)}
$$


Proposition 1.5 implies the existence of $\theta$-thick sequences for all nonnegative functions $\theta(t)$.

For simplicity of notation, we write $\rho_{1}=\rho_{\theta_{1}}, \rho_{2}=\rho_{\theta_{2}}$.

Proposition 1.6. Assume that $\rho_{1}(t)$ satisfies the conditions of Proposition 1.5. (1.4) holds and $\rho_{1}(t) / \rho_{2}(t) \rightarrow \infty$ as $t \rightarrow 0$. Then $\Lambda_{\theta_{1}} \neq \Lambda_{\theta_{2}}$. Moreover, there exists a sequence $\left\{z_{k}\right\}$ such that $\left\{z_{k}\right\} \notin \Lambda_{\theta_{1}}$, but (1.2) with $\rho_{\theta}=\rho_{2}$ holds for every $f \in H^{\infty}$.

Notice that the sufficient part of Proposition 1.5 (the existence of a $\theta$-thick sequence when (1.4) is satisfied) follows from Proposition 1.6. taking $\theta_{1}=\theta$ and an arbitrary $\theta_{2}$.

In Section 2 we establish some preliminary results. Some of them are of independent interest. For example, Proposition 2.3 gives conditions on $\left\{z_{k}\right\}$ which imply that $\left\{z_{k}\right\}$ is $\theta$-thick, as well as a sufficient condition of existence of $\theta$-thin sequences. We develop these results in Section 5 .

\section{Some SUfFICIENT AND SOME NECESSARY CONDITIONS}

First, we would like to show that our problem, which is a priori given in terms of infinite sums, reduces in many cases to a problem about the rate of decrease of $|f(z)|$ as a function of $(1-|z|)$, when $z$ is restricted to the sequence $\left\{z_{k}\right\}$. Such problems have already been investigated in $[\mathrm{PT}]$ and numerous other works, see the references in $[\mathrm{EE}$.

Proposition 2.1. (a) Suppose that $\left\{z_{k}\right\} \in \Lambda_{\theta}, \theta(t) \geq 0$. Then there exists a non identically vanishing function $f_{1} \in H^{\infty}(\mathbb{D})$ such that

$$
\left|f_{1}\left(z_{k}\right)\right| \leq e^{-\theta\left(1-\left|z_{k}\right|\right)}
$$

for all $k \in \mathbb{Z}_{+}$.

(b) Conversely, suppose that

$$
\liminf _{t \rightarrow 0} \frac{\theta(t)}{\log \log \frac{1}{t}}=: L>0,
$$

that $\left\{z_{k}\right\}$ is separated, non-Blaschke, and that there exists a function $f \in H^{\infty}(\mathbb{D})$ such that

$$
\left|f\left(z_{k}\right)\right| \leq e^{-\theta\left(1-\left|z_{k}\right|\right)}
$$

for all $k \in \mathbb{Z}_{+}$. Then $\left\{z_{k}\right\} \in \Lambda_{\theta}$.

Proof. (a) There exists a non identically vanishing function $f \in H^{\infty}(\mathbb{D})$ such that $\sum_{k}\left(1-\left|z_{k}\right|\right) e^{\theta\left(1-\left|z_{k}\right|\right)}\left|f\left(z_{k}\right)\right|<\infty$. Define

$$
\left\{z_{j}^{\prime}\right\}:=\left\{z_{k}:\left|f\left(z_{k}\right)\right| \leq e^{-\theta\left(1-\left|z_{k}\right|\right)}\right\} .
$$

Then

$$
\sum_{k: z_{k} \notin\left\{z_{j}^{\prime}\right\}}\left(1-\left|z_{k}\right|\right) \leq \sum_{k: z_{k} \notin\left\{z_{j}^{\prime}\right\}}\left(1-\left|z_{k}\right|\right) e^{\theta\left(1-\left|z_{k}\right|\right)}\left|f\left(z_{k}\right)\right|<\infty .
$$

So $\left\{z_{k}\right\} \backslash\left\{z_{j}^{\prime}\right\}$ is a Blaschke sequence. Let $B$ denote the Blaschke product with zeroes on $\left\{z_{k}\right\} \backslash\left\{z_{j}^{\prime}\right\}$, then $f_{1}:=f B$ clearly satisfies the desired conclusion.

(b) Let

$$
Y_{n}:=\left\{z: 1-2^{-n} \leq|z|<1-2^{-n-1}\right\}
$$


Since $\left\{z_{k}\right\}$ is separated, $\sum_{k: z_{k} \in Y_{n}}\left(1-\left|z_{k}\right|\right) \leq C_{\delta}$. Let $m \in \mathbb{Z}_{+}$to be chosen later, $f_{1}:=f^{m}$. Then, because of the above and of the hypothesis, for any $\varepsilon>0$,

$$
\begin{aligned}
\sum_{k}\left(1-\left|z_{k}\right|\right) e^{\theta\left(1-\left|z_{k}\right|\right)}\left|f\left(z_{k}\right)^{m}\right| & \leq \sum_{n} \sum_{k: z_{k} \in Y_{n}}\left(1-\left|z_{k}\right|\right) e^{-(m-1) \theta\left(1-\left|z_{k}\right|\right)} \\
\leq & C_{\varepsilon}+C_{\delta} \sum_{n} e^{-(m-1)(L-\varepsilon) \log n}=C_{\varepsilon}+C_{\delta} \sum_{n} \frac{1}{n^{(m-1)(L-\varepsilon)}}<\infty
\end{aligned}
$$

for $m$ large enough (for $L=\infty$, replace $L-\varepsilon$ by any positive number).

Proof of the necessity part in Theorem 1.3.

The existence of a non-trivial $f_{1} \in H^{\infty}(\mathbb{D})$ such that $\left|f_{1}(z)\right| \leq e^{-\theta(1-|z|)}$ over the sequence $\left\{z_{k}\right\}$, which is non-Blaschke and separated, implies that $g(t):=e^{-\theta(1-t)}$ is not an essential minorant in the language of [LS]. The main result of that paper is that $g$ is an essential minorant if and only if

$$
\int^{1} \frac{d t}{(1-t) \log \left(\frac{1}{g(t)}\right)}<\infty
$$

which is exactly the negation of (1.3). Therefore, if (1.3) does not hold, there are no sequences in $\Lambda_{\theta}$.

For a given sequence $\left\{z_{i}\right\}$ let $N_{m}$ be a number of points $z_{i}$ in $Y_{m}$, where $Y_{m}$ is as in (2.1).

Lemma 2.2. Let $\sum_{m} N_{m} 2^{-m}=\infty$. Then for every $f \in H^{\infty}, f \not \equiv 0$, there exists a set $\mathcal{J}$ of indices with the following properties: $\sum_{m \in \mathcal{J}} N_{m} 2^{-m}<\infty$, and

$$
\left|f\left(z_{k}\right)\right|>\exp \left\{-C(f, \delta) 2^{m} / N_{m}\right\} \quad \text { for at least } N_{m} / 2 \text { points } z_{k} \in Y_{m}, m \notin \mathcal{J},
$$

where $C(f, \delta)$ is a positive constant and $\delta$ is the constant from (1.1).

Lemma 2.2 was in fact proved in [EE] (see proof of Proposition 7.9 (a)), although it was not formulated in this explicit form. In Section 4 we give another, essentially self-contained proof (without resorting to Govorov and Grishin as in [EE]).

Proposition 2.3. Let $\theta(t)$ be a positive nonincreasing function and $\rho_{\theta}(t)=t e^{\theta(t)}$.

a) Suppose that $\left\{z_{k}\right\}$ is a separated sequence and

$$
\sum_{m \notin \mathcal{J}} N_{m} \rho_{\theta}\left(2^{-m}\right) \exp \left\{-\gamma 2^{m} / N_{m}\right\}=\infty \quad \text { for every } \gamma>0
$$

and for every set $\mathcal{J}$ of indices with $\sum_{m \in \mathcal{J}} N_{m} 2^{-m}<\infty$. Then $\left\{z_{k}\right\} \notin \Lambda_{\theta}$.

b) Suppose that a set $\mathcal{L}$ of indices and a sequence $\left\{N_{m}\right\}$ of nonnegative integers are such that $N_{m} \uparrow \infty, N_{m} 2^{-m} \downarrow 0$ as $m \in \mathcal{L}, m \rightarrow \infty$, and

$$
\sum_{m \in \mathcal{L}} N_{m} \rho_{\theta}\left(2^{-m}\right) \exp \left\{-\gamma 2^{m} / N_{m}\right\}<\infty \quad \text { for some } \gamma>0 \text {. }
$$

Then there exists a separated $\theta$-thin sequence $\left\{z_{k}\right\}$, for which $N_{m}$ are the given numbers. 


\section{Examples}

a) If $N_{m}=p_{m} 2^{m} /\left(\theta\left(2^{-m}\right)+\log m\right)$, where $p_{m} \rightarrow \infty$, then $\left\{z_{k}\right\} \notin \Lambda_{\theta}$.

b) If $N_{m}=p 2^{m} /\left(\theta\left(2^{-m}\right)+\log m\right)$ with a positive constant $p$, then there exists a separated sequence $\left\{z_{k}\right\} \in \Lambda_{\theta}$, for which $N_{m}$ are the given numbers.

Proposition 2.3 generalizes and refines Proposition 7.9 in EE]. Its proof will be given in Section 4

\section{Proofs of the main Results}

Proof of Theorem 1.4 (a). It will be enough to show that if $\theta_{1} \geq c \theta_{2}$, for some $c>0$, then $\Lambda_{\theta_{1}} \subset \Lambda_{\theta_{2}}$. Without loss of generality, assume that $c<1$.

Suppose that $\left\{z_{k}\right\} \in \Lambda_{\theta_{1}}$. By Proposition [2.1, we can pick a non identically vanishing function $f_{1} \in H^{\infty}(\mathbb{D})$ such that

$$
\left|f_{1}\left(z_{k}\right)\right| \leq e^{-\theta_{1}\left(1-\left|z_{k}\right|\right)}, \quad \forall k \in \mathbb{Z}_{+} .
$$

Pick an integer $m \geq 1+c^{-1}$. Then

$$
\left|f_{1}\left(z_{k}\right)^{m}\right| \leq e^{-c^{-1} \theta_{1}\left(1-\left|z_{k}\right|\right)} e^{\theta_{1}\left(1-\left|z_{k}\right|\right)}\left|f_{1}\left(z_{k}\right)\right|
$$

So

$$
\begin{aligned}
\sum_{k} e^{\theta_{2}\left(1-\left|z_{k}\right|\right)}\left|f_{1}\left(z_{k}\right)^{m}\right| & \leq \sum_{k} e^{\theta_{2}\left(1-\left|z_{k}\right|\right)-c^{-1} \theta_{1}\left(1-\left|z_{k}\right|\right)} e^{\theta_{1}\left(1-\left|z_{k}\right|\right)}\left|f_{1}\left(z_{k}\right)\right| \\
& \leq \sum_{k} e^{\theta_{1}\left(1-\left|z_{k}\right|\right)}\left|f_{1}\left(z_{k}\right)\right|<\infty
\end{aligned}
$$

so $f_{1}^{m}$ is a non identically vanishing function in $H^{\infty}(\mathbb{D})$ which proves that $\left\{z_{k}\right\} \in \Lambda_{\theta_{2}}$.

Proof of Theorem 1.4 (b). Assume that (1.3) holds with $\theta=\theta_{2}$. First we construct a sequence $\left\{z_{k}\right\} \in \Lambda_{\theta_{2}}$.

We note that (1.3) is equivalent to the following condition:

$$
\sum_{m=1}^{\infty}\left[\theta_{2}\left(2^{-m}\right)\right]^{-1}=\infty .
$$

Without loss of generality we may assume that $\theta_{2}(t) \uparrow \infty$ as $t \rightarrow 0$. We show that there exist a set $\mathcal{L}$ of indices and a nondecreasing sequence $\left\{N_{m}\right\}$ of positive integers with the following properties:

$$
\begin{gathered}
\sum_{m \in \mathcal{L}}\left[\theta_{2}\left(2^{-m}\right)\right]^{-1}=\infty, \quad \sum_{m \in \mathcal{L}}\left[\theta_{2}\left(2^{-m}\right)\right]^{-2}<\infty \\
2^{m-1} / \theta_{2}\left(2^{-m}\right)<N_{m} \leq 2^{m} / \theta_{2}\left(2^{-m}\right), \quad N_{m} 2^{-m} \downarrow 0 \text { as } m \in \mathcal{L}, m \rightarrow \infty .
\end{gathered}
$$

Denote $\varepsilon_{m}=\left[\theta_{2}\left(2^{-m}\right)\right]^{-1}$. Then $\varepsilon_{m} \downarrow 0$ and $\sum_{m} \varepsilon_{m}=\infty$. Choose integers $m_{n}, M_{n}$ such that $m_{n}<M_{n} \leq m_{n+1}$ and

$$
\frac{1}{2 n}<\sum_{m=m_{n}+1}^{M_{n}} \varepsilon_{m}<\frac{1}{n},
$$


and set $\mathcal{L}_{1}=\bigcup_{n}\left\{m_{n}+1, \ldots, M_{n}\right\}$. Then (3.2) holds with $\mathcal{L}=\mathcal{L}_{1}$. Let

$$
\mathcal{J}_{m}=\left\{j: j>m, 2^{j} \varepsilon_{j} \leq 2^{m} \varepsilon_{m}\right\}, \quad \mathcal{L}=\mathcal{L}_{1} \backslash \bigcup_{m \in \mathcal{L}_{1}} \mathcal{J}_{m}
$$

Since

$$
\sum_{j \in \mathcal{J}_{m}} \varepsilon_{j} \leq 2^{m} \varepsilon_{m} \sum_{j=m+1}^{\infty} 2^{-j}=\varepsilon_{m}
$$

the relations (3.2) with $\mathcal{L}=\mathcal{L}_{1}$ imply the same relations with $\mathcal{L}$. For $m, k \in \mathcal{L}$, $m<k$ we have $2^{m-k} \varepsilon_{m}<\varepsilon_{k}<\varepsilon_{m}$. Fix $m_{1} \in \mathcal{L}$ and set $N_{m_{1}}=\left[2^{m_{1}} \varepsilon_{m_{1}}\right]$, where [.] denotes the integral part of a number. Suppose that $N_{m}$ is already defined, and $k=\min \{j: j \in \mathcal{L}, j>m\}$. Then we set

$$
N_{k}=\min \left\{2^{k-m} N_{m},\left[2^{k} \varepsilon_{k}\right]\right\} .
$$

Since $2^{m} \varepsilon_{m}<2^{k} \varepsilon_{k}$, we have $N_{k} \geq N_{m}$.

Now we prove (3.3). If $N_{k}=\left[2^{k} \varepsilon_{k}\right]$, then the inequalities in (3.3) are evident. If $N_{k}=2^{k-m} N_{m}$, then by induction we have

$$
2^{k-1} \varepsilon_{k}<2^{k-1} \varepsilon_{m}=2^{k-m} 2^{m-1} \varepsilon_{m}<2^{k-m} N_{m}=N_{k} .
$$

Moreover, the inequality $N_{k} \leq N_{m} 2^{k-m}$ implies that $N_{k} 2^{-k} \leq N_{m} 2^{-m}$. Thus, (3.3) is established.

Let $l_{m}=N_{m} 2^{-m}$. By (3.3) we have $\theta_{2}\left(2^{-m}\right) \leq 1 / l_{m}<2 \theta_{2}\left(2^{-m}\right)$, and

$$
\begin{aligned}
N_{m} \rho_{2}\left(2^{-m}\right) \exp \left\{-\gamma 2^{m} / N_{m}\right\} & =l_{m} \exp \left\{\theta_{2}\left(2^{-m}\right)-\gamma / l_{m}\right\} \\
& \leq l_{m} \exp \left\{-1 / l_{m}\right\}<l_{m}^{2},
\end{aligned}
$$

if $\gamma$ is sufficiently big. By (3.2),$\sum_{m \in \mathcal{L}} l_{m}^{2}<\infty$. Thus, the condition (2.3) is satisfied, and the existence of a separated $\theta_{2}$-thin sequence $\left\{z_{k}\right\}$ follows from Proposition 2.3 $\mathrm{b}$ ).

Now we show that $\left\{z_{k}\right\} \notin \Lambda_{\theta_{1}}$. Let $p_{m}=\theta_{1}\left(2^{-m}\right) / \theta_{2}\left(2^{-m}\right)$. By (3.3) $), \theta_{2}\left(2^{-m}\right) \leq$ $2^{m} / N_{m}<2 \theta_{2}\left(2^{-m}\right)$. For $\rho_{1}(t)=t e^{\theta_{1}(t)}$ and for a fixed constant $\gamma$ we have

$$
\begin{aligned}
N_{m} \rho_{1}\left(2^{-m}\right) \exp \left\{-\gamma 2^{m} / N_{m}\right\} & =l_{m} \exp \left\{\theta_{1}\left(2^{-m}\right)-\gamma 2^{m} / N_{m}\right\} \\
& \geq l_{m} \exp \left\{\theta_{2}\left(2^{-m}\right)\left(p_{m}-2 \gamma\right)\right\}>l_{m}
\end{aligned}
$$

for sufficiently big $m \in \mathcal{L}$. By (3.2) and (3.3) $\sum_{m \in \mathcal{L}} l_{m}=\infty$. We see that (2.2) holds, and by Proposition 2.3 a) $\left\{z_{k}\right\} \notin \Lambda_{\theta_{1}}$.

Proof of the necessity part of Proposition 1.5.

Suppose that (1.4) fails. By taking a Riemann sum, we will show that any separated sequence is thin, even better : that we can take as function $f$ the constant 1 . The separation condition (1.1) implies that every Whitney square contains at most $M=$ $M(\delta)$ points $z_{k}$. Hence, $N_{m}=O\left(2^{m}\right)$ for every separated sequence $\left\{z_{k}\right\}$. Then

$$
\sum_{k} \rho\left(1-\left|z_{k}\right|\right) \leq \sum_{m} N_{m} \rho\left(2^{-m}\right) \leq C \sum_{m} 2^{m} \rho\left(2^{-m}\right) ;
$$

the convergence of the last series is equivalent to the negation of (1.4).

To prove Proposition [1.6, we need the following elementary fact about numerical series. 
Lemma 3.1. If $\sum_{n} 2^{n} \rho_{1}\left(2^{-n}\right)=\infty, \rho_{1}(t) \leq C t$ and $\rho_{1}(t) / \rho_{2}(t) \rightarrow \infty$ as $t \rightarrow 0$, we can choose a subset of the integers $\mathcal{A} \subset \mathbb{Z}_{+}$such that

$$
\sum_{n \in \mathcal{A}} 2^{n} \rho_{2}\left(2^{-n}\right)<\infty
$$

and

$$
\sum_{n \in \mathcal{A}} 2^{n} \rho_{1}\left(2^{-n}\right)=\infty
$$

Proof. We can recursively define a strictly increasing sequence of integers $\left\{n_{j}\right\}$ such that

(1) $\rho_{2}\left(2^{-n}\right) \leq 2^{-j} \rho_{1}\left(2^{-n}\right), n \geq n_{j}$;

(2) $\sum_{n_{j} \leq n<n_{j+1}} 2^{n} \rho_{1}\left(2^{-n}\right) \geq 1$.

Now we pick

$$
n_{j}^{\prime}:=\min \left\{m \geq n_{j}: \sum_{n_{j} \leq n \leq m} 2^{n} \rho_{1}\left(2^{-n}\right) \geq 1\right\}
$$

and set

$$
\mathcal{A}_{j}:=\left\{n \in \mathbb{Z}_{+}: n_{j} \leq n \leq n_{j}^{\prime}\right\}, \quad \mathcal{A}:=\cup_{j} \mathcal{A}_{j} .
$$

It is clear that $\sum_{\mathcal{A}} 2^{n} \rho_{1}\left(2^{-n}\right) \geq \sum_{j} 1=\infty$. Because $2^{n} \rho_{1}\left(2^{-n}\right) \leq C$ for all $n$, $\sum_{\mathcal{A}_{j}} 2^{n} \rho_{1}\left(2^{-n}\right) \leq 1+C$ for all $j$, therefore by $(1)$

$$
\sum_{\mathcal{A}} 2^{n} \rho_{2}\left(2^{-n}\right) \leq \sum_{j} \sum_{\mathcal{A}_{j}} 2^{n} \rho_{2}\left(2^{-n}\right) \leq \sum_{j} 2^{-j}(1+C)<\infty .
$$

Proof of Proposition 1.6. Consider the sequence $S=\left\{z_{m, j}: m \in \mathcal{A}, 0 \leq j<2^{m}\right\}$, where

$$
z_{m, j}:=\left(1-2^{-m}\right) \exp \left(2^{-m} 2 \pi i j\right), \quad 0 \leq j<2^{m},
$$

and $\mathcal{A}$ is the set from Lemma 3.1. For any $f \in H^{\infty}$, by (3.4),

$$
\sum_{z \in S} \rho_{2}(1-|z|)|f(z)| \leq\|f\|_{\infty} \sum_{z \in S} \rho_{2}(1-|z|)=\|f\|_{\infty} \sum_{n \in \mathcal{A}} 2^{n} \rho_{2}\left(2^{-n}\right)<\infty .
$$

Now we will show that $S \notin \Lambda_{\theta_{1}}$. Let $f \in H^{\infty}, f \not \equiv 0$, be given, and let $\mathcal{J}$ be the set from Lemma 2.2. Since $N_{m}=2^{m}$ as $m \in \mathcal{A}$, the set $\mathcal{J}$ is finite, and by (3.5) we have

$$
\sum_{k} \rho_{1}\left(1-\left|z_{k}\right|\right)\left|f\left(z_{k}\right)\right| \geq \frac{1}{2} \sum_{m \in \mathcal{A} \backslash \mathcal{J}} 2^{m} \rho_{1}\left(2^{-m}\right) \exp \{-C(f, \delta)\}=\infty
$$




\section{Proof of Lemma 2.2 and Proposition 2.3.}

Our alternative prove of Lemma 2.2 is based on the following Lemma, which is proved in [NPT, p. 124, lines 3 to 17] and is the main ingredient in [PT, Lemma 3].

Lemma A. Let $f \in H^{\infty}(\mathbb{D})$ and $\delta^{\prime} \in(0,1)$. Then there exists a function $h$, positive and harmonic on $\mathbb{D}$, such that for all $z$ for which $d_{G}\left(z, f^{-1}(0)\right) \geq \delta^{\prime}$,

$$
e^{-h(z)} \leq|f(z)|
$$

In particular, if $\left\{z_{k}\right\}$ is a separated sequence, there exists a Blaschke sequence $b \subset\left\{z_{k}\right\}$ such that for any $z_{k} \notin b$,

$$
e^{-h\left(z_{k}\right)} \leq\left|f\left(z_{k}\right)\right|
$$

Proof of Lemma 2.2. By Lemma A, there exists a positive harmonic function $h$ and a Blaschke sequence $b \subset\left\{z_{k}\right\}$ such that

$$
\text { For } z_{k} \notin b, e^{-h\left(z_{k}\right)}<\left|f\left(z_{k}\right)\right| \text {. }
$$

Let

$$
\mathcal{J}:=\left\{m: \# b \cap Y_{m} \geq N_{m} / 4\right\} .
$$

Since $b$ is a Blaschke sequence, we have $\sum_{m \in \mathcal{J}} N_{m} 2^{-m}<\infty$.

Let $C(f, \delta)>0$ be a constant to be chosen later. For a point $a \in Y_{m}$, let $I_{a} \subset \partial \mathbb{D}$ be the arc centered at $a /|a|$, of length $2^{-m}$. By the separation condition, the $I_{z_{k}}$ for $z_{k} \in Y_{m}$ form a covering of finite multiplicity of $\partial \mathbb{D}$, that is to say, there exists a positive constant $C_{1}(\delta)$ such that

$$
\sum_{z_{k} \in Y_{m}} \chi_{I_{z_{k}}} \leq C_{1}(\delta)
$$

By Harnack's inequality, for $\theta$ such that $e^{i \theta} z_{k} /\left|z_{k}\right| \in I_{z_{k}}$, we have

$$
h\left(e^{i \theta}\left(1-2^{-m}\right) z_{k} /\left|z_{k}\right|\right) \geq C_{2} h\left(z_{k}\right) .
$$

For $m \in \mathcal{J}$, let

$$
N_{m}^{\prime}:=\#\left\{z_{k} \in Y_{m} \backslash b: h\left(z_{k}\right)>C(f, \delta) 2^{m} / N_{m}\right\} .
$$

The mean-value property for harmonic functions gives

$$
h(0)=\int_{0}^{2 \pi} h\left(\left(1-2^{-m}\right) e^{i \theta}\right) \frac{d \theta}{2 \pi} \geq C_{1}(\delta)^{-1} \cdot N_{m}^{\prime} \cdot 2^{-m} \cdot C_{2} C(f, \delta) 2^{m} / N_{m}
$$

therefore

$$
N_{m}^{\prime} \leq\left(C_{2} C(f, \delta)\right)^{-1} C_{1}(\delta) h(0) N_{m}<N_{m} / 4
$$

for $C(f, \delta)$ large enough. At the remaining points $z_{k}$, of which there are at least $N_{m} / 2$, we do have

$$
\left|f\left(z_{k}\right)\right| \geq e^{-h\left(z_{k}\right)} \geq \exp \left\{-C(f, \delta) 2^{m} / N_{m}\right\} .
$$

Proof of Proposition 2.3 (a). Under conditions of Proposition 2.3, we have

$$
\sum_{m} N_{m} 2^{-m}=\infty
$$


Moreover, $\rho_{\theta}(1-|z|)=(1-|z|) e^{\theta(1-|z|)}>2^{-m-1} e^{\theta\left(2^{-m}\right)}=\frac{1}{2} \rho_{\theta}\left(2^{-m}\right), z \in Y_{m}$. Let $f \in H^{\infty}, f \not \equiv 0$, be given, and let $\mathcal{J}$ be the set from Lemma 2.2. If $\gamma>C(f, \delta)$, then by $(2.2)$

$$
\begin{aligned}
\sum_{k} \rho_{\theta}\left(1-\left|z_{k}\right|\right)\left|f\left(z_{k}\right)\right| & \geq \frac{1}{2} \sum_{m \notin \mathcal{J}} \sum_{z_{k} \in Y_{m}} \rho_{\theta}\left(2^{-m}\right)\left|f\left(z_{k}\right)\right| \\
& \geq \frac{1}{4} \sum_{m \notin \mathcal{J}} N_{m} \rho_{\theta}\left(2^{-m}\right) \exp \left\{-\gamma 2^{m} / N_{m}\right\}=\infty
\end{aligned}
$$

Lemma 4.1. Suppose that $P_{1}(t), t \in(0,1)$, is a nondecreasing function, $P_{1}(t)(1-t)$ is a nonincreasing function tending to 0 as $t \rightarrow 1$, and $d_{m} \leq 1$ are given positive numbers. Then there are a sequence $\left\{z_{k}\right\} \subset \mathbb{D}$ and an absolute constant $M$ with the following properties: all points $z_{k}$ are situated on the circumferences $|z|=1-2^{-m}$, $m=1,2, \ldots$;

$$
\begin{gathered}
\min \left\{\left|z_{j}-z_{k}\right|: j \neq k,\left|z_{j}\right|=\left|z_{k}\right|=1-2^{-m}\right\} \geq d_{m} ; \\
N_{z}\left(1-2^{-m}\right)>M\left(d_{m} P_{1}\left(1-2^{-m}\right)\right)^{-1}+1, \quad m=1,2, \ldots,
\end{gathered}
$$

where $N_{z}\left(1-2^{-m}\right)$ is the number of points $z_{k}$ with $\left|z_{k}\right|=1-2^{-m}$, and for every $T>0$ there is a nontrivial function $f \in H^{\infty}$ such that

$$
\log \left|f\left(z_{k}\right)\right| \leq-P_{1}\left(\left|z_{k}\right|\right) T, \quad k=1,2 \ldots
$$

We omit the proof, which is exactly the same as the proof of Lemma 4.3, part 2 in [EE] with $\phi\left(t_{j}\right)=1 / d_{j}$ and $t_{j}=1-2^{-j}$. It follows from Lemma 4.3 in [EE] that Lemma 4.1 is sharp.

Proof of Proposition 2.3 (b). Pick $d_{m}=2^{-m}$. By assumption, $N_{m} 2^{-m} \downarrow 0,\left\{N_{m}\right\}$ is a nondecreasing sequence and $N_{m} \rightarrow \infty$ on $\mathcal{L}$. Hence, there exists a nondecreasing function $P_{1}(t)$ such that

$$
P_{1}\left(1-2^{-m}\right)=M 2^{m} / N_{m}, \quad m \in \mathcal{L},
$$

where $M$ is the constant from (4.1), $P_{1}(t) \uparrow \infty, P_{1}(t)(1-t) \downarrow 0$ as $t \rightarrow 1$. Let $\left\{z_{k}\right\}$ be the sequence from Lemma 4.1. By (4.1),

$$
N_{z}\left(1-2^{-m}\right)>N_{m}, \quad m \in \mathcal{L} .
$$

If we choose a sufficiently big number $T$, then by (4.2)

$$
\log \left|f\left(z_{k}\right)\right| \leq-M 2^{m} T / N_{m}<-\gamma 2^{m} / N_{m}, \quad\left|z_{k}\right|=1-2^{-m}, \quad m \in \mathcal{L} .
$$

For every $m \in \mathcal{L}$ we choose $N_{m}$ points from $\left\{z_{k}\right\}$ with $\left|z_{k}\right|=1-2^{-m}$. We also denote this subsequence by $\left\{z_{k}\right\}$. Using (2.3), we have

$$
\begin{aligned}
\sum_{k} \rho_{\theta}\left(1-\left|z_{k}\right|\right)\left|f\left(z_{k}\right)\right| & =\sum_{m \in \mathcal{L}} \sum_{z_{k} \in Y_{m}} \rho_{\theta}\left(1-\left|z_{k}\right|\right)\left|f\left(z_{k}\right)\right| \leq \\
& \leq c \sum_{m \in \mathcal{L}} N_{m} \rho_{\theta}\left(2^{-m}\right) \exp \left\{-\gamma 2^{m} / N_{m}\right\}<\infty .
\end{aligned}
$$

Thus, $\left\{z_{k}\right\} \in \Lambda_{\theta}$, and the proof of Proposition 2.3 is complete. 


\section{Further PROPERTIES OF THIN SEQUENCES}

The following assertion was proved in [EE] (see Proposition 7.8). Let $N_{m}$ be given nonnegative integers, and let

$$
z_{m, k}=\left(1-2^{-m}\right) \exp \left(2 \pi i k / N_{m}\right), \quad 1 \leq k \leq N_{m}, \quad m=1,2, \ldots
$$

for $N_{m}>0$. Then $\left\{z_{m, k}\right\} \notin \Lambda_{\theta}$ for $\rho_{\theta} \equiv 1$ if and only if $\left\{z_{m, k}\right\}$ is a non-Blaschke sequence, that is $\sum_{m} N_{m} 2^{-m}=\infty$.

On the other hand, if we know only, that $\left\{z_{m, k}\right\}$ is a separated sequence, we need essentially stronger conditions on $\left\{N_{m}\right\}$ (see Proposition 2.3 with $\rho_{\theta} \equiv 1$ ). Thus, there is a certain connection between the dispersion of points over annuluses $Y_{m}$ and the massivity of a sequence, i. e. numbers $N_{m}$ (we also can interpret this relation as a connection between the dispersion of points and the possible decay of nontrivial bounded function over a sequence). In the present section we investigate this connection. In particular, we develop Lemma 2.2. Proposition 2.3 and some results in [EE], and give another approach to obtaining such assertions, which is based on estimates of subharmonic functions outside exceptional sets.

Let a sequence $\left\{z_{k}\right\}$ be given. For every $m$, for which $Y_{m}$ contains at least six points $z_{k}$, and for every $z_{k} \in Y_{m}$ we set

$$
d_{m, k}:=\min \left\{\left|z_{j}-z_{k}\right|: j \neq k, z_{j}, z_{k} \in Y_{m}\right\} .
$$

Let $\bar{d}_{m}$ be the mean value of $\left[N_{m} / 6\right]$ smallest numbers $d_{m, k}$ (here [.] is the integral part of a number). Our main tool is the following lemma.

Lemma 5.1. Let $\left\{z_{k}\right\}$ be a separated sequence. Let a set $\mathcal{K}$ of indices be such that $N_{m} \geq 6$ as $m \in \mathcal{K}$, and $\sum_{m \in \mathcal{K}} N_{m} 2^{-m}=\infty$. Then for every $f \in H^{\infty}, f \neq \equiv$, there exists a set $\mathcal{J}$ of indices with the following properties: $\sum_{m \in \mathcal{J}} N_{m} 2^{-m}<\infty$, and

$$
\left|f\left(z_{k}\right)\right|>\exp \left\{-C(f, \delta)\left(N_{m} \bar{d}_{m}\right)^{-1}\right\}
$$

for at least $N_{m} / 2$ points $z_{k} \in Y_{m}$, when $m \in \mathcal{K} \backslash \mathcal{J}$, where $C(f, \delta)$ is a positive constant and $\delta$ is the constant from (1.1).

Since $\bar{d}_{m}>$ Const $2^{-m}$, Lemma 5.1 refines Lemma 2.2. We shall prove Lemma 5.1 in Section 6].

Corollary 5.2. Let $\theta(t)$ be a positive nonincreasing function and $\rho_{\theta}(t)=t e^{\theta(t)}$.

a) Suppose that $\left\{z_{k}\right\}$ is a separated sequence. Let $\mathcal{K}$ be a set of indices satisfying conditions of Lemma 5.1 and such that

$$
\sum_{m \in \mathcal{K} \backslash \mathcal{J}} N_{m} \rho_{\theta}\left(2^{-m}\right) \exp \left\{-\gamma\left(N_{m} \bar{d}_{m}\right)^{-1}\right\}=\infty \quad \text { for every } \gamma>0
$$

and for every set $\mathcal{J}$ of indices with $\sum_{m \in \mathcal{J}} N_{m} 2^{-m}<\infty$. Then $\left\{z_{k}\right\} \notin \Lambda_{\theta}$.

b) Suppose that a set $\mathcal{L}$ of indices, a sequence $\left\{N_{m}\right\}$ of nonnegative integers and a sequence $\left\{d_{m}\right\}$ of positive numbers are such that $1 \geq d_{m} \geq 2^{-m},\left\{d_{m} 2^{m}\right\}$ is a nondecreasing sequence, $N_{m} \uparrow \infty, N_{m} d_{m} \downarrow 0$ as $m \in \mathcal{L}, m \rightarrow \infty$, and

$$
\sum_{m \in \mathcal{L}} N_{m} \rho_{\theta}\left(2^{-m}\right) \exp \left\{-\gamma\left(N_{m} d_{m}\right)^{-1}\right\}<\infty \quad \text { for some } \gamma>0 \text {. }
$$

Then there exists a separated $\theta$-thin sequence $\left\{z_{k}\right\}$ with $\left|z_{k}\right|=1-2^{-m}$ as $z_{k} \in Y_{m}$, for which $N_{m}$ are the given numbers and $\bar{d}_{m} \geq d_{m}$. 
Proof. (a) The proof is essentially the same as the proof of Proposition 2.3 (a) with the obvious corrections: we use Lemma 5.1 instead of Lemma 2.2.

(b) We argue by analogy with the proof of Proposition 2.3 (b). Let $P_{1}(t)$ be a nondecreasing function such that $P_{1}(t) \uparrow \infty, P_{1}(t)(1-t) \downarrow 0$ as $t \rightarrow 1$ and

$$
P_{1}\left(1-2^{-m}\right)=M\left(N_{m} d_{m}\right)^{-1}, \quad m \in \mathcal{L},
$$

where $M$ is the constant from (4.1). Let $\left\{z_{k}\right\}$ be the sequence from Lemma 4.1. By (4.1),

$$
N_{z}\left(1-2^{-m}\right) \geq N_{m}, \quad m \in \mathcal{L} .
$$

By (4.2), for sufficiently big numbers $T$ we have

$$
\log \left|f\left(z_{k}\right)\right| \leq-M T\left(N_{m} d_{m}\right)^{-1}<-\gamma\left(N_{m} d_{m}\right)^{-1}, \quad\left|z_{k}\right|=1-2^{-m}, \quad m \in \mathcal{L} .
$$

For every $m \in \mathcal{L}$ we choose $N_{m}$ points from $\left\{z_{k}\right\}$ with $\left|z_{k}\right|=1-2^{-m}$. The obtained sequence we also denote by $\left\{z_{k}\right\}$. Using (5.3), we have

$$
\begin{aligned}
\sum_{k} \rho_{\theta}\left(1-\left|z_{k}\right|\right)\left|f\left(z_{k}\right)\right| & =\sum_{m \in \mathcal{L}} \sum_{z_{k} \in Y_{m}} \rho_{\theta}\left(1-\left|z_{k}\right|\right)\left|f\left(z_{k}\right)\right| \leq \\
& \leq c \sum_{m \in \mathcal{L}} N_{m} \rho_{\theta}\left(2^{-m}\right) \exp \left\{-\gamma\left(N_{m} d_{m}\right)^{-1}\right\}<\infty .
\end{aligned}
$$

Corollary 5.2 shows the connection between the dispersion of points over annuluses $Y_{m}$ and the massivity of a sequence $\left\{z_{k}\right\}$. It is clear that Corollary 5.2 is a direct generalization of Proposition 2.3. Another special case gives the following assertion.

Corollary 5.3. Let $N_{m} \bar{d}_{m} \geq c>0$ as $m \in \mathcal{K}$ and $\rho_{\theta}(t) \geq c_{1} t, c_{1}>0$. Then a separated sequence $\left\{z_{k}\right\}$ is $\theta$-thick if and only if

$$
\sum_{m \in \mathcal{K}} N_{m} 2^{-m}=\infty
$$

Proof. Clearly, if $\left\{z_{k}\right\} \notin \Lambda_{\theta}$, then (5.4) holds.

Conversely, the condition (5.4) and the inequality $\rho_{\theta}(t) \geq c_{1} t$ imply (5.2), and our assertion follows from Corollary 5.2 .

Corollary 5.3 is a generalization of Proposition 7.8 in $\mathrm{EE}$, quoted at the beginning of this section. It is easy to see that Corollary 5.3 is not correct for $\rho_{\theta}(t)$ such that $\liminf _{t \rightarrow 0} \rho_{\theta}(t) / t=0$. Indeed, in this case there exist numbers $t_{j} \in(0,1)$ for which $t_{j+1} / t_{j}<1 / 2$ and $\sum_{j=1}^{\infty} \rho_{\theta}\left(t_{j}\right) / t_{j}<\infty$. Let $\mathcal{K}=\left\{m_{j}\right\}$, where $2^{-m_{j}-1}<t_{j} \leq 2^{-m_{j}}$, and let $N_{m_{j}}=2^{m_{j}}, z_{m_{j}, k}=\left(1-t_{j}\right) \exp \left(2 \pi i k / N_{m_{j}}\right), 1 \leq k<N_{m_{j}}$. Then $\left\{z_{m_{j}, k}\right\}$ is a separated $\theta$-thin sequence satisfying (5.4).

\section{Proof of Lemma 5.1.}

We need certain preliminary definitions and results. Let $f$ be a meromorphic function in $\mathbb{D}$, and let $\left\{a_{k}\right\}$ and $\left\{b_{k}\right\}$ be zeros and poles of $f$, respectively. We assume 
that 0 is neither a zero nor a pole of $f$. The Nevanlinna characteristic of $f$ is defined by (cf. for example Hayman [Ha], p. 4)

$$
T_{f}(r)=\frac{1}{2 \pi} \int_{0}^{2 \pi} \log ^{+}\left|f\left(r e^{i \varphi}\right)\right| d \varphi+\sum_{\left|b_{i}\right|<r} \log \frac{r}{\left|b_{i}\right|} .
$$

Since $T_{f}(r)$ is a nondecreasing function of $r, \lim _{r \uparrow 1} T_{f}(r)=: T_{f}(1)$ exists for functions $f$ in the Nevanlinna class $N$, i. e., functions of bounded characteristic.

To $f \in H^{\infty}(\mathbb{D})$ with zeros $\left\{a_{n}\right\}, a_{n} \neq 0$, we associate a non-negative measure $\eta$ on the closed unit disc $\overline{\mathbb{D}}$ defined as follows: for every set $E \subset \overline{\mathbb{D}}$ for which $E \cap \partial \mathbb{D}$ is measurable, we define

$$
\eta(E)=\lim _{r \rightarrow 1} \frac{1}{2 \pi} \int_{e^{i \theta} \in E} \log ^{-}\left|f\left(r e^{i \theta}\right)\right| d \theta+\sum_{a_{n} \in E} \log \frac{1}{\left|a_{n}\right|} .
$$

We note that $\eta(\overline{\mathbb{D}})=T_{1 / f}(1)$. For the proof that this limit exists, we refer to $[\mathrm{EE}$, Appendix 1]. We quote the following theorem in the form given in [EE] (see Theorem 2.10 in [EE] and the remark after it).

Theorem 6.1. (Govorov-Grishin) Suppose that $f \in H^{\infty}(\mathbb{D}), f(z) \not \equiv 0$. For every $P>1$ there exists a system of discs $D^{k}\left(w_{k}, r_{k}\right)$ such that

$$
\begin{gathered}
\log |f(z)|>-A_{1} P T_{1 / f}(1), \quad z \in \mathbb{D} \backslash \bigcup_{k} D^{k}, \\
\sum_{k} r_{k}<1 / P,
\end{gathered}
$$

and every $z \in \bigcup_{k} D^{k}$ belongs to at most $A_{2}$ discs $D^{k}$ (we shall say that $A_{2}$ is the multiplicity of this covering). Moreover,

$$
\eta\left(D^{k}\right)>A_{3} P T_{1 / f}(1) r_{k} .
$$

Here $A_{1}, A_{2}$ and $A_{3}$ are absolute positive constants and $\eta$ is the non-negative measure on the closed unit disc $\overline{\mathbb{D}}$ defined by (6.1).

Proof of Lemma 5.1. Let

$$
\begin{aligned}
\mathcal{M}_{0}=\left\{m: N_{m} \bar{d}_{m}>1 / 2\right\} & \\
\mathcal{M}_{j}=\left\{m: 2^{-j-1}<N_{m} \bar{d}_{m} \leq 2^{-j}\right\}, j & =1,2, \ldots, \\
G_{j} & =\bigcup_{m \in \mathcal{M}_{j}} Y_{m}, \quad P_{j}=c(\delta) 2^{j},
\end{aligned}
$$

where $c(\delta)$ is a positive constant which will be chosen later, depending only on $\delta$, and $\delta$ is the constant from (1.1). Let a function $f \in H^{\infty}, f \neq \equiv 0$, be given and let $D_{j}^{k}\left(w_{j, k}, r_{j, k}\right)$ be a system of disks from Theorem 6.1 with $P=P_{j}$. We introduce the 
following sets of disks:

$$
\begin{array}{lll}
\mathcal{D}_{j, 1}=\left\{D_{j}^{k}: D_{j}^{k} \cap G_{j} \neq \varnothing,\right. & \left.r_{j, k}<\frac{1}{8}\left(1-\left|w_{j, k}\right|\right)\right\}, & \mathcal{D}_{1}=\bigcup_{j} \mathcal{D}_{j, 1} ; \\
\mathcal{D}_{j, 2}=\left\{D_{j}^{k}: D_{j}^{k} \cap G_{j} \neq \varnothing,\right. & \left.r_{j, k} \geq \frac{1}{8}\left(1-\left|w_{j, k}\right|\right)\right\}, & \mathcal{D}_{2}=\bigcup_{j} \mathcal{D}_{j, 2} .
\end{array}
$$

We shall show that

$$
\sum_{z_{l} \in \mathcal{D}_{1}}\left(1-\left|z_{l}\right|\right)<\infty
$$

By (1.1), each disk $D_{j}^{k} \in \mathcal{D}_{1}$ contains at most $N=N(\delta)$ points $z_{l}$. Moreover, disks from $\mathcal{D}_{1}$ do not intersect $\partial \mathbb{D}$, and by (6.1)

$$
\eta\left(D_{j}^{k}\right)=\sum_{a_{i} \in D_{j}^{k}} \log \frac{1}{\left|a_{i}\right|}, \quad D_{j}^{k} \in \mathcal{D}_{1},
$$

where $a_{i}$ are zeros of $f$. By (6.4),$\eta\left(D_{j}^{k}\right)>0$. Hence, every disc $D_{j}^{k} \in \mathcal{D}_{1}$ contains at least one zero of $f$. Each disk in $\mathcal{D}_{1}$ intersects at most two sets $Y_{m}$. For each $j$ the multiplicity of the family $\left\{D_{j}^{k}\right\}$ is the absolute constant $A_{2}$. Hence, the multiplicity of the covering $\mathcal{D}_{1}$ is at most $3 A_{2}$. Thus,

$$
\begin{aligned}
\sum_{z_{l} \in \mathcal{D}_{1}}\left(1-\left|z_{l}\right|\right) \leq \sum_{D_{j}^{k} \in \mathcal{D}_{1}} \sum_{z_{l} \in D_{j}^{k}}\left(1-\left|z_{l}\right|\right) \leq \\
\quad \leq c \sum_{D_{j}^{k} \in \mathcal{D}_{1}} \sum_{a_{i} \in D_{j}^{k}}\left(1-\left|a_{i}\right|\right) \leq 3 A_{2} c \sum_{i}\left(1-\left|a_{i}\right|\right)<\infty,
\end{aligned}
$$

that what we need.

Let $\mathcal{J}_{1}$ be the set of indices $m$ such that $\#\left\{z_{l}: z_{l} \in Y_{m} \cap \mathcal{D}_{1}\right\} \geq N_{m} / 6$. Then

$$
\sum_{m \in \mathcal{J}_{1}} N_{m} 2^{-m} \leq c \sum_{z_{l} \in \mathcal{D}_{1}}\left(1-\left|z_{l}\right|\right)<\infty
$$

We set $\mathcal{K}_{1}=\mathcal{K} \backslash \mathcal{J}_{1}$. Then at least $\frac{5}{6} N_{m}$ points $z_{l}$ do not belong to $\mathcal{D}_{1}$ for every $m \in \mathcal{K}_{1}$.

Fix $j \geq 0$. We split the points $z_{l} \in \mathcal{D}_{j, 2} \cap G_{j}$, into two subsets $Z_{j, 1}$ and $Z_{j, 2}$ in the following way. Let $z_{l} \in Y_{m}$. Then $z_{l} \in Z_{j, 1}$, if every disk $D_{j}^{k} \in \mathcal{D}_{j, 2}$ containing $z_{l}$, does not contain other points $z_{l^{\prime}} \in Y_{m} ; z_{l} \in Z_{j, 2}$, if there exists a disk $D_{j}^{k} \in \mathcal{D}_{j, 2}$ containing at least one other point $z_{l^{\prime}} \in Y_{m}$ besides $z_{l}$. Let

$$
\begin{aligned}
N_{m}^{j, i}=\#\left\{z_{l}: z_{l} \in Y_{m} \cap Z_{j, i}\right\}, \quad & i=1,2 ; \\
& \mathcal{J}_{j, 2}=\left\{m \in \mathcal{M}_{j}: N_{m}^{j, 1} \geq N_{m} / 6\right\}, \quad \mathcal{J}_{2}=\bigcup_{j} \mathcal{J}_{j, 2} .
\end{aligned}
$$

We shall prove that

$$
\sum_{m \in \mathcal{J}_{2}} N_{m} 2^{-m}<\infty
$$

Assume that $D_{j}^{k} \in \mathcal{D}_{j, 2}$ and $D_{j}^{k} \cap Y_{m} \neq \varnothing$. Then

$$
r_{j, k}>\frac{1}{9} 2^{-m-1} \text {. }
$$


Indeed, if $r_{j, k} \leq \frac{1}{9} 2^{-m-1}$, then by the inequality $\left|w_{j, k}\right|<1-2^{-m-1}+r_{j, k}$ we have

$$
r_{j, k} \geq \frac{1}{8}\left(1-\left|w_{j, k}\right|\right)>\frac{1}{8}\left(2^{-m-1}-r_{j, k}\right) \geq \frac{1}{8} \cdot 8 r_{j, k}=r_{j, k},
$$

and we come to a contradiction. From (6.5) we deduce that

$$
\sum_{m: D_{j}^{k} \cap Y_{m} \neq \varnothing} 2^{-m}<2 \cdot 2 \cdot 9 r_{j, k}=36 r_{j, k} .
$$

Moreover, the number of disks $D_{j}^{k} \in \mathcal{D}_{j, 2}$ intersecting $Y_{m}$ is at least $N_{m}^{j, 1}$. Hence,

$$
\begin{aligned}
\sum_{m \in \mathcal{J}_{j, 2}} N_{m} 2^{-m} \leq 6 \sum_{m \in \mathcal{J}_{j, 2}} & N_{m}^{j, 1} 2^{-m} \\
& \leq 6 \sum_{k: D_{j}^{k} \in \mathcal{D}_{j, 2}} \sum_{m: D_{j}^{k} \cap Y_{m} \neq \varnothing} 2^{-m}<6 \cdot 36 \sum_{k} r_{j, k}<\frac{c}{P_{j}}=c_{1} 2^{-j}
\end{aligned}
$$

(in the last inequality we used (6.3)). We have

$$
\sum_{m \in \mathcal{J}_{2}} N_{m} 2^{-m}=\sum_{j} \sum_{m \in \mathcal{J}_{j, 2}} N_{m} 2^{-m}<c \sum_{j} 2^{-j}<\infty .
$$

Now we shall show that

$$
N_{m}^{j, 2} \leq \frac{1}{6} N_{m} \text { for all } m \in \mathcal{K} \cap \mathcal{M}_{j}
$$

For fixed $m$ we consider disks $D_{j}^{k}$ containing more than one point $z_{l} \in Y_{m} \cap Z_{j, 2}$. Then $2 r_{j, k}>d_{m, l}$. The separation condition (1.1) implies that $d_{m, l} \geq c(\delta) 2^{-m}$, where $\delta$ is the constant from (1.1); and since the disks $D\left(z_{l}, d_{m, l} / 2\right)$ are disjoint, denoting by $\lambda_{2}$ the area measure,

$$
\begin{aligned}
C(\delta) 2^{-m} r_{j, k} & \geq \lambda_{2}\left(D\left(w_{j, k}, 2 r_{j, k}\right) \cap Y_{m}\right) \\
& \geq \sum_{l: z_{l} \in D_{j}^{k} \cap Y_{m} \cap Z_{j, 2}} \lambda_{2}\left(D\left(z_{l}, d_{m, l} / 2\right) \cap Y_{m}\right) \\
& \geq c^{\prime}(\delta) 2^{-m} \sum_{l: z_{l} \in D_{j}^{k} \cap Y_{m} \cap Z_{j, 2}} d_{m, l} .
\end{aligned}
$$

Thus

$$
\sum_{l: z_{l} \in D_{j}^{k} \cap Y_{m} \cap Z_{j, 2}} d_{m, l}<c_{2}(\delta) r_{j, k} .
$$

If (6.6) does not hold, then

$$
\begin{aligned}
\frac{1}{6} N_{m} \bar{d}_{m}< & N_{m}^{j, 2} \bar{d}_{m} \leq \sum_{l: z_{l} \in Y_{m} \cap Z_{j, 2}} d_{m, l} \\
& \leq \sum_{k} \sum_{l: z_{l} \in D_{j}^{k} \cap Y_{m} \cap Z_{j, 2}} d_{m, l} \leq \sum_{k} c_{2}(\delta) r_{j, k}<\frac{c_{2}(\delta)}{P_{j}}=\frac{c_{2}(\delta)}{c(\delta)} 2^{-j}<\frac{1}{6} N_{m} \bar{d}_{m},
\end{aligned}
$$

if $c(\delta)$ is sufficiently big. That contradiction proves the validity of (6.6) . Thus, if $\mathcal{J}=\mathcal{J}_{1} \cup \mathcal{J}_{2}$, then for every $m \in \mathcal{K} \backslash \mathcal{J}$ the number of points $z_{l}$ lying outside of $\bigcup_{k} D_{j}^{k}$ is at least

$$
\frac{5}{6} N_{m}-N_{m}^{j, 1}-N_{m}^{j, 2}>\frac{5}{6} N_{m}-\frac{1}{6} N_{m}-\frac{1}{6} N_{m}=\frac{1}{2} N_{m}
$$


The inequality (5.1) follows from (6.2).

\section{REFERENCES}

[CTW] Y. S. Chow, T. Trent and J. Li-Ming Wang, "A separating problem on function spaces", J. Math. Anal. Appl., 111 (1985), 177-187.

[E1] V. Ya. Eiderman, "Measure and capacity of exceptional sets arising in estimations of $\delta$ subharmonic functions", in Potential Theory, edited by M. Kishi, Walter de Gruyter : Berlin, New York, 1991, 171-177

[E2] V. Ya. Eiderman, "On the sum of values of functions in certain classes on a sequence of points" (Russian), Izv. Vyssh. Uchebn. Zaved. Mat. No. 1 (1992), 89-97.

[E3] V. Ya. Eiderman, "Approximation by polynomials with small coefficients" (Russian), Mat. Zametki 57, No. 1 (1995), 150-153. English transl. in Math. Notes 57, No. 1-2 (1995), 110-113.

[EE] V. Ya. Eiderman and M. Essén, "Uniqueness theorems for analytic and subharmonic functions" (Russian), Algebra i Analiz 14, No. 6 (2002), 1-88. English transl. in St. Petersburg Math. J. 14, No. 6 (2003).

[Ha] W. K. Hayman, Meromorphic functions, Clarendon Press : Oxford, 1964.

$[\mathrm{Kh}] \quad$ S. Ya. Khavinson, "On a notion of completeness taking into account the values of coefficients of approximating polynomials", Izv. Akad. Nauk Arm. SSR, Ser. Mat. 6, No. 2-3 (1971), 221-234.

[LS] Yu. I. Lyubarskii and K. Seip, "A uniqueness theorem for bounded analytic functions", Bull. London Math. Soc. 29 (1997), 49-52.

[NPT] A. Nicolau, J. Pau and P. J. Thomas, "Smallness sets for bounded holomorphic functions", J. d'Analyse Math. 82 (2000), 119-148.

[Ni] N. K. Nikolski, "Complete extensions of Volterra operators" (Russian), Izv. Akad. Nauk SSSR, Ser. Mat., 33 (1969), 1349-1355.

[PT] J. Pau and P. J. Thomas, "Decrease of bounded holomorphic functions along discrete sets", Proceedings of the Edinburgh Mathematical Society, to appear; electronically available from math arXiv : http://arXiv.org/abs/math.CV/0106048

Vladimir Ya. Eiderman, Moscow State Civil Engineering University, 129337 Moscow, Yaroslavskoe shosse, 26, Russia.

eiderman@orc.ru

Pascal J. Thomas, Laboratoire de Mathématiques Emile Picard, UMR CNRS 5580, Université Paul Sabatier, 118 route de Narbonne, 31062 Toulouse CEDEX, France. pthomas@cict.fr 